\title{
Clarithromycin attenuates autophagy in myeloma cells
}

\author{
MIKI NAKAMURA ${ }^{1}$, YOSHITAKA KIKUKAWA ${ }^{1}$, MOTOHIRO TAKEYA ${ }^{2}$, \\ HIROAKI MITSUYA ${ }^{1}$ and HIROYUKI HATA ${ }^{1}$ \\ ${ }^{1}$ Department of Hematology, Kumamoto University Hospital; ${ }^{2}$ Department of Cell Pathology, \\ Graduate School of Medical Sciences, Kumamoto University, 1-1-1 Honjo, Kumamoto 860-8556, Japan
}

Received April 20,2010; Accepted June 18, 2010

DOI: 10.3892/ijo_00000731

\begin{abstract}
It has been reported that clarithromycin (CAM) augments the anti-tumor activity of thalidomide against multiple myeloma (MM) cells, while the mechanism remains unclear. A myeloma cell line or primary myeloma cells were treated with CAM. Autophagy was analyzed by morphological changes, LC3 expression and lysotracker staining. CAM induced vacuoles in the cytoplasm of MM cells which resembled autolysosomes. The manifestation of the CAMinduced vacuoles was blocked by treatment with PI3-kinase inhibitor. CAM induced an accumulation of LC3-II without affecting the mTOR or AKT pathways, eventually leading to cell death. CAM may halt the autophagy process after fusion of autophagosomes with lysosomes. This phenomenon may explain how CAM, combined with thalidomide, augments the cytotoxic effects of the latter on MM cells and suggests that modification of autophagy might represent a new approach for therapy of MM.
\end{abstract}

\section{Introduction}

Certain macrolide antibiotics are known to exert effects other than anti-bacterial activity, such as growth inhibition of certain cancer cells. Prolonged survival of lung cancer patients treated with macrolides, and experiments showing anti-tumor activity of CAM have been reported (1-5). In experimental settings, various effects of macrolides have been reported, e.g., anti-angiogenesis (6) and direct antitumor activity (7). Clarithromycin (CAM) is known to exert a potent anti-tumor effect on multiple myeloma cells, when combined with thalidomide or dexamethasone (8), despite there being some controversy at a setting of mono-therapy (9). Of note, high efficacy to myeloma of chemotherapeutic regimen combining CAM with lenalidomide, a derivative of thalidomide, has recently been reported (10).

Correspondence to: Dr Hiroyuki Hata, Department of Hematology, Kumamoto University Hospital, 1-1-1 Honjo, Kumamoto 860-8556, Japan

E-mail: hata@kumamoto-u.ac.jp

Key words: autophagy, macrolide antibiotics, multiple myeloma
In an attempt to clarify the mechanisms regulating the anti-tumor activity of CAM, we analyzed its direct effect on myeloma cells in vitro. We showed significant induction of vacuoles in human myeloma cell lines and primary myeloma cells by the treatment with CAM, which are related to morphological features of autophagy. Detailed analysis revealed that inhibition of autophagy process at late stage by CAM may contribute augmentation of anti-myeloma effect. We believe that this report is the first one showing a contribution of CAM to autophagy. Moreover, this finding may lead to a novel therapeutic approach by manipulating autophagy system, which has been proposed, but not yet clinically achieved (11).

\section{Materials and methods}

Cells and cell culture. The myeloma cell line 12PE (12), and primary myeloma cells purified by CD138-conjugated immuno-magnetic beads (Miltenvi Biotec, Auburn, CA, USA) were utilized. Clarithromycin (CAM) was obtained from Taisho-Toyama Pharmaceuticals (Tokyo, Japan) and dissolved in DMSO at a concentration of $10 \mathrm{mg} / \mathrm{ml}$ as a stock solution. Cells were cultured in RPMI-1640 medium supplemented with $10 \%$ fetal calf serum (Sigma, St. Louis, MO, USA) at $37^{\circ} \mathrm{C}$ under humidified conditions.

Analysis of autophagy. Morphology was analyzed either by May-Giemsa staining or electron microscopy. Lysosomes were stained with LysoTracker (Invitrogen, Carlsbad, CA, USA) and analyzed using fluorescent microscopy. Antibodies to LC3 were a kind gift of Dr T. Yoshimori (Department of Cellular Regulation, Research Institute for Microbial Diseases, Osaka University). Antibodies to phospho-AKT, AKT and p-70S6K1 were purchased from Santa Cruz Biotechnology (Santa Cruz, CA, USA). Western blotting was performed by utilizing a Pre-cast Gel kit (Invitrogen), and membranes were visualized by ECL Plus detection kit (GE Healthcare Bio-Sciences, Uppsala, Sweden). 3-methyladenine, LY294002 and Bafilomycin A (BAF) were purchased from Sigma. Microscopic analysis for LysoTracker staining, a dye that stains acidic lysosomes, was performed with confocal laser scanning microscopy (Olympus, Tokyo, Japan).

Electron microscopy. Cell pellets were fixed with $1.0 \%$ glutaraldehyde for $60 \mathrm{~min}$ and postfixed with $1.0 \%$ osmium 
A
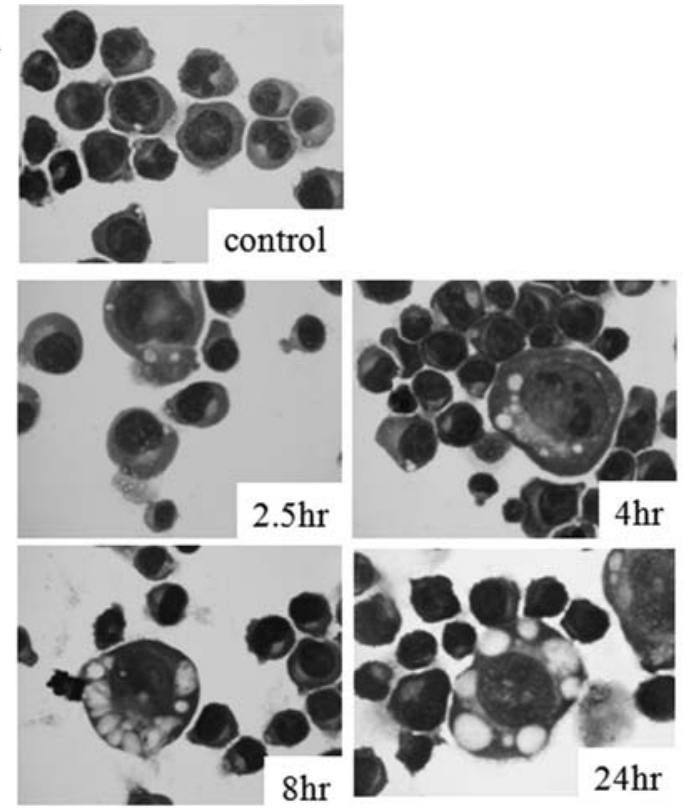

B

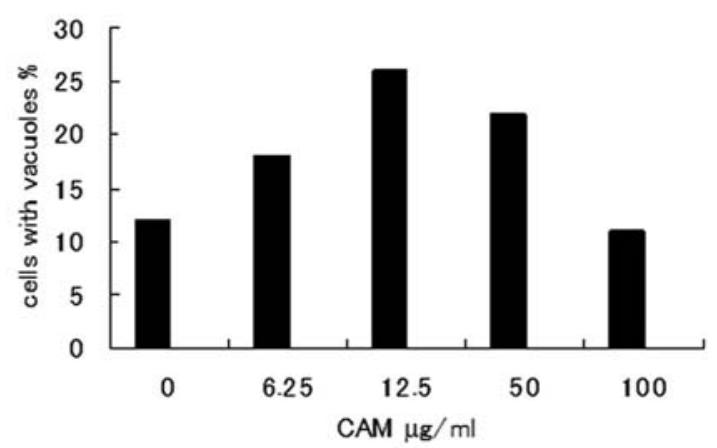

Figure 1. Induction of vacuoles in the cytoplasm of myeloma cells by CAM. (A) Time-dependent induction of vacuoles by CAM. Myeloma cell line 12PE was incubated with CAM at $50 \mu \mathrm{g} / \mathrm{ml}$ for the indicated periods. Significant induction of vacuoles was found in a time-dependent manner. (B) Dose-dependent induction of vacuoles by CAM. 12PE cells were treated with CAM at indicated concentrations for $18 \mathrm{~h}$. Cells were stained with May-Giemsa staining solution on cytospin slides, and at least 300 cells were counted by microscopy. The proportions of cells with vacuoles are shown.

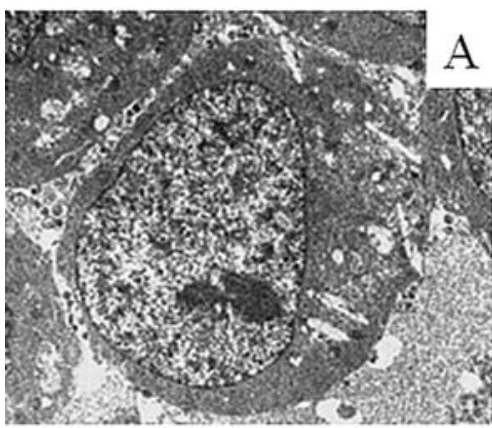

control

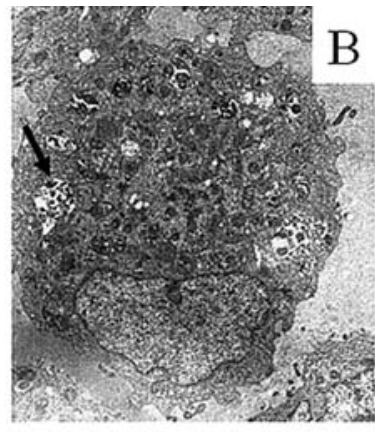

CAM

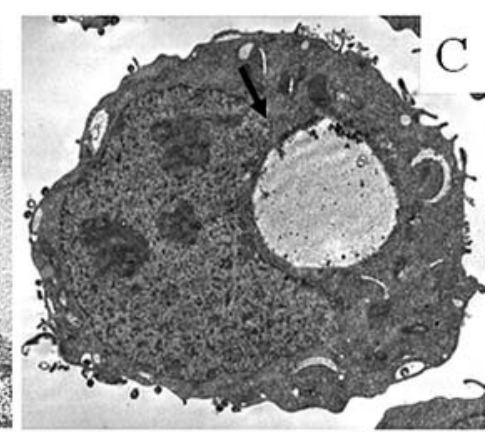

CAM

Figure 2. Electron microscopic analysis of 12PE cells treated with CAM at $50 \mu \mathrm{g} / \mathrm{ml}$ for $18 \mathrm{~h}$. (A) Untreated cell; (B and C) CAM treated cells. Vacuoles with degenerated organelle considered to be autophagic vacuoles (B, arrow). Large vacuole with empty structure considered to be autolysosomes (C, arrow).

tetroxide for $30 \mathrm{~min}$ at $4^{\circ} \mathrm{C}$. After dehydration in a graded ethanol series, the cells were embedded in epoxy resin. Ultrathin sections stained with uranyl acetate and lead citrate were observed using an H-7500 electron microscope (Tokyo, Japan).

Flow cytometry. Cells were stained with the Annexin V/PI staining kit (Medical and Biological Laboratory, Nagoya, Japan). Cells were then analyzed by Epics V flow cytometer (Coulter, Miami, FL, USA).

\section{Results}

Induction of vacuoles by CAM. The myeloma cell line 12PE was incubated with CAM at various concentrations for $18 \mathrm{~h}$. Vacuolization was morphologically observed after treatment with CAM at a concentration of $50 \mu \mathrm{g} / \mathrm{ml}$, and increased in a time-dependent manner with maximum vacuolization at $12.5 \mu \mathrm{g} / \mathrm{ml}$ which is potentially achievable concentration within cytoplasm (Fig. 1A). Quantification of vacuoles revealed that CAM increased vacuolization in a dose-dependent manner (Fig. 1B). Electron microscopy revealed that the small vacuoles represented vacuoles that contained degenerated organelles (Fig. 2B) and larger vacuoles containing no organelles (Fig. 2C) which are considered to be autophagosomes and autolysosomes, respectively. Primary purified myeloma cells cultured with CAM at a concentration of $10 \mu \mathrm{g} / \mathrm{ml}$ for $18 \mathrm{~h}$ showed similar vacuole formation to that found in cell lines (Fig. 3).

Co-localization of vacuoles with lysosomes. Electron microscopy suggested that small vacuoles are autophagosomes, 


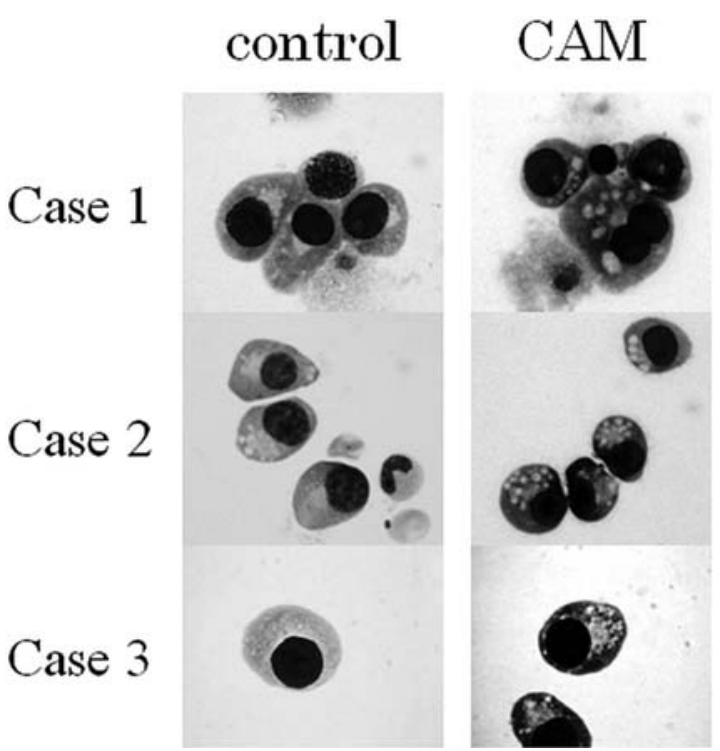

Figure 3. Induction of vacuoles by CAM in freshly isolated myeloma cells. Samples were obtained from three myeloma patients and purified by immunomagnetic beads. Cells were incubated with or without CAM at $10 \mu \mathrm{g} / \mathrm{ml}$ for $18 \mathrm{~h}$. Cells were stained with May-Giemsa solution. Purified myeloma cells from all cases showed marked induction of vacuoles in the cytoplasm, as found in the myeloma cell line 12PE. therefore, we hypothesized that large vacuoles observed by light microscopy may have been autolysosomes. To confirm this hypothesis, 12PE cells were treated with CAM and stained with LysoTracker, a dye that stains acidic lysosomes. As shown in Fig. 4, large vacuoles were stained red with LysoTracker, which clearly indicated that the vacuoles were acidic lysosomes. When cells were treated with CAM at increasing doses, vacuoles stained with LysoTracker accumulated in a dose-dependent manner (Fig. 5). This finding was also confirmed in primary purified myeloma cells (Fig. 6).

CAM induces accumulation of LC3-II. Taking these results together, it is suggested that CAM-induced vacuoles are autophagy-related vacuoles. To further examine this possibility, we analyzed processing of LC3-I to LC3-II, a hallmark of autophagy. We found that more accumulation of LC3-II by CAM as concentration of CAM increased (Fig. 7A). These results indicate either induction of autophagy or inhibition of autophagy at late stage by CAM. Subsequently, phosphorylated and total Akt were analyzed, since autophagy is initiated by dephosphorylation of Akt (13). However, phosphorylated Akt was not inhibited by CAM (Fig. 7B), suggesting that CAM may not induce autophagy through dephospholyration of AKT.
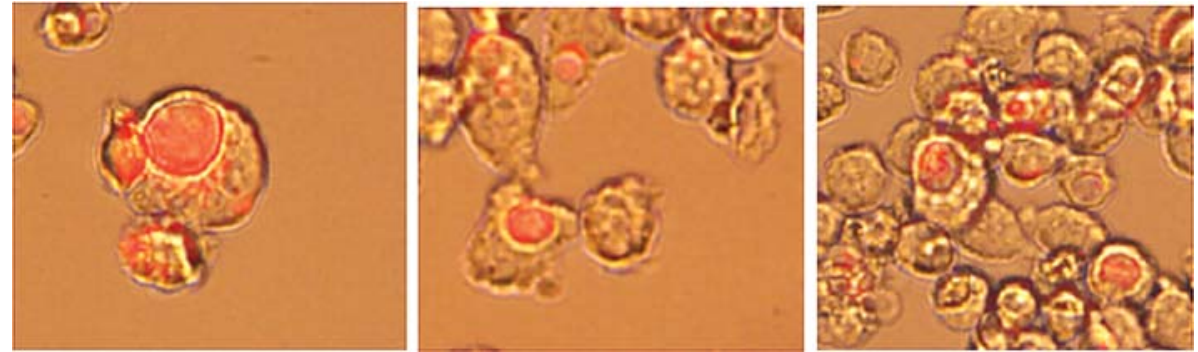

Figure 4. LysoTracker staining merged with phase contrast microscopy. $12 \mathrm{PE}$ cells were treated with CAM at $50 \mu \mathrm{g} / \mathrm{ml}$ for $18 \mathrm{~h}$. Cells were then stained with LysoTracker for $15 \mathrm{~min}$. Images of fluorescent microscopy and phase contrast microscopy were merged. Note that the red stain of LysoTracker co-localizes with large vacuoles.
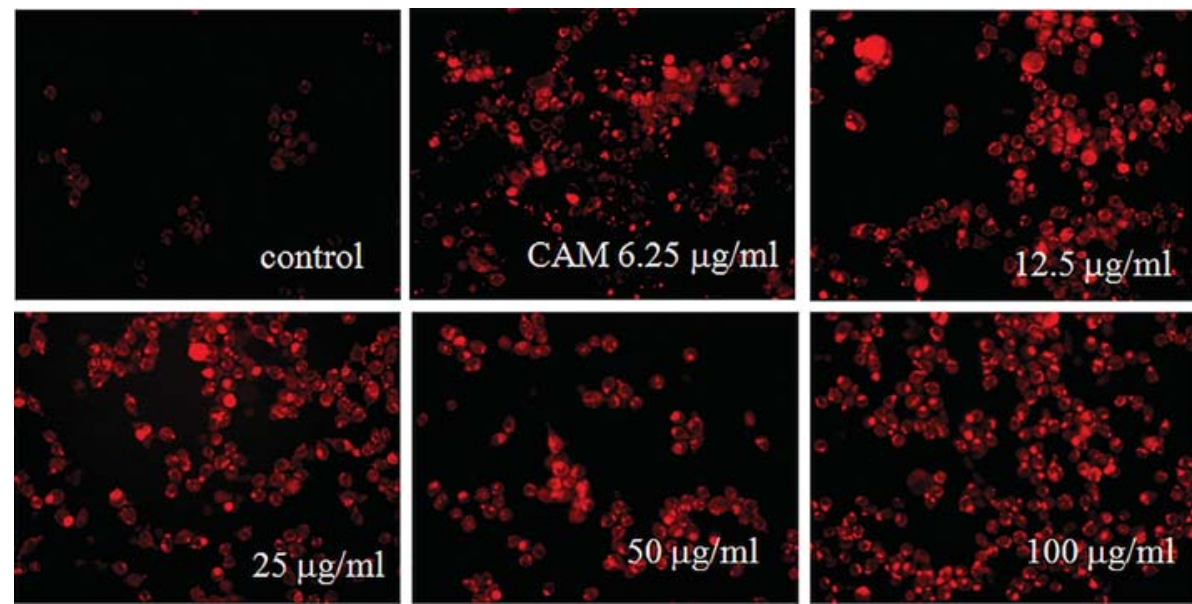

Figure 5. Induction of LysoTracker-positive vacuoles by CAM. 12PE cells were treated with CAM at indicated concentrations for $18 \mathrm{~h}$. Vacuoles stained with LysoTracker accumulated by CAM in a dose-dependent manner. 

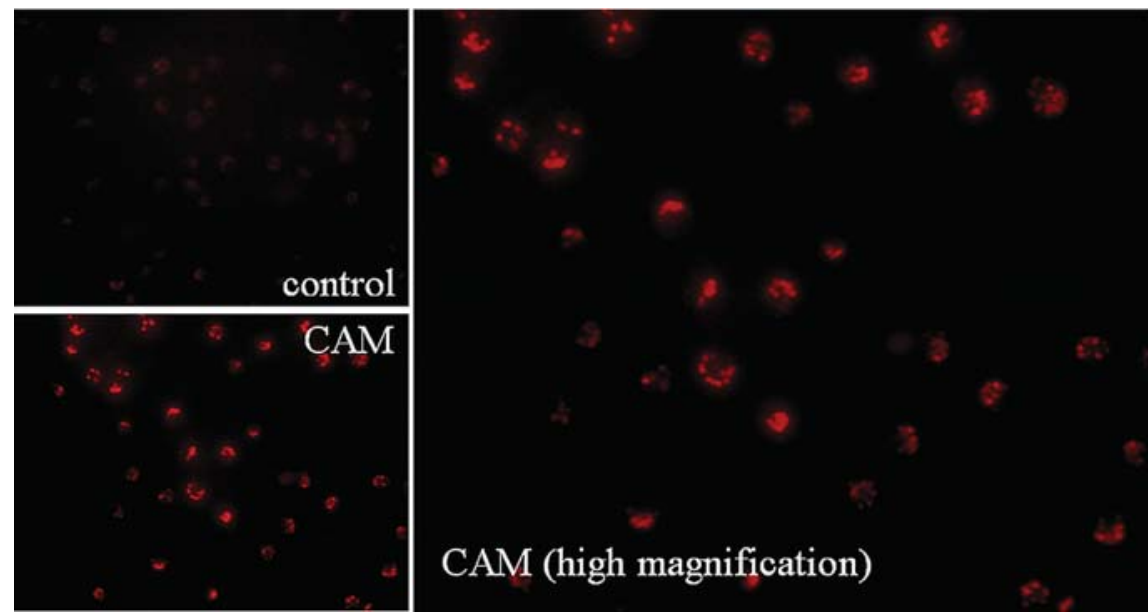

Figure 6. Induction of LysoTracker staining by CAM in freshly isolated myeloma cells. Myeloma cells from patient bone marrow were purified with immunomagnetic beads and incubated with CAM at $10 \mu \mathrm{g} / \mathrm{ml}$ for $18 \mathrm{~h}$. Cells were then stained with LysoTracker. Induction of LysoTracker staining was clearly observed following treatment with CAM.

A

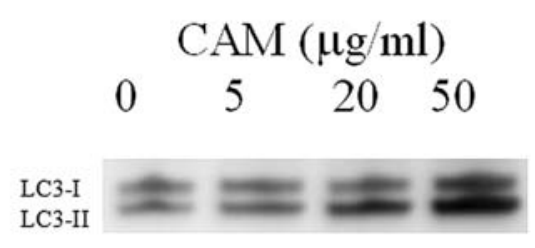

B

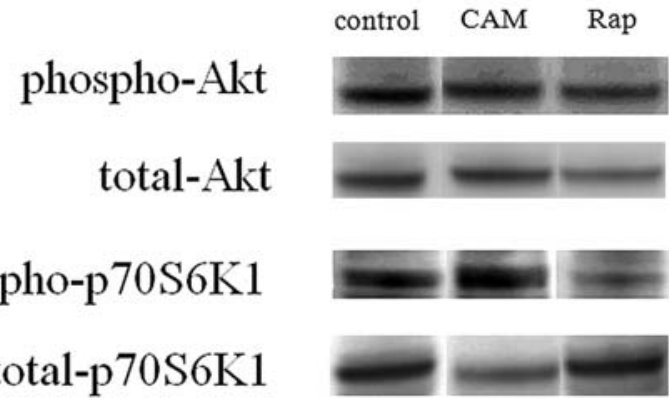

Figure 7. Induction of LC3-II and inhibition of phospho-p70S6K by CAM. $12 \mathrm{PE}$ cells were treated with CAM at $50 \mu \mathrm{g} / \mathrm{ml}$ for $18 \mathrm{~h}$. Cell lysates were analyzed by Western blotting. (A) Dose-dependent induction of LC3-II was observed. (B) No reduction of phospho-p70S6K1 and phosphorylated AKT were found following treatment with CAM. Significant inhibition of phosho$\mathrm{P} 70 \mathrm{~S} 6 \mathrm{~K} 1$ by rapamycin (Rap) at $1 \mu \mathrm{M}$ is shown as a positive control.

Inhibition of vacuolization by 3-methyladenine. We further examined p70S6K1 since it locates downstream of mTOR, which is known to inhibit autophagy $(14,15)$. There was no reduction of phospholyrated p70S6K by CAM (Fig. 7B) while rapamycin, an inhibitor of mTOR, markedly decreased phospho-p70S6K. These results suggest that CAM is not involved in regular autophagy initiation process mediated by mTOR inhibition.

We further treated cells with autophagy inhibitor 3-methyladenine (3MA), an inhibitor of autophagy-initiation, in combination with CAM. Interestingly, vacuole formation by CAM was clearly inhibited by $3 \mathrm{MA}$, as shown by morpho- logical analysis (Fig. 8A) and LysoTracker staining (Fig. 8B), suggesting that CAM-induced vacuole formation locates downstream of autophagy initiation.

Induction of cytotoxicity by CAM. Since the combination of CAM and thalidomide is reported to be effective for myeloma $(8,10)$, we considered that CAM alone might affect cell viability. To analyze this possibility, 12PE cells were treated with CAM and analyzed by Annexin V/PI staining. As shown in Fig. 9, CAM induced necrotic cell death or late apoptosis, which is shown by double positivity for Annexin V and PI. Since CAM induced vacuolization of cytoplasm, which is distinct from fragmentation of nucleus, and no significant induction of caspase 3 by CAM (data not shown), CAM induced necrotic cell death rather than apoptosis.

\section{Discussion}

Autophagy is a phenomenon that starts with autophagosome formation and leads to fusion of autophagosomes with lysosomes (16). It is recognized that the initiation of autophagy is induced by nutrient starvation. Although a direct and simple method for identifying autophagy has not been established, specific morphology under the electron microscope and processing of LC3-I to LC3-II are well recognized as features of autophagy (17). As these processes are controlled by $3 \mathrm{MA}$ (18), inhibition of vacuoles by $3 \mathrm{MA}$ also confirms that vacuoles are related to autophagy.

As we found induction of small vacuolization following treatment with CAM that led to large vacuole formation we investigated the possible relation of autophagy with CAM, and found that CAM indeed induced autophagic vacuoles, which was proven by morphological examination with electron microscopy and detection of LC3-II. Induction of autophagic vacuoles by CAM was further confirmed by treating cells with $3 \mathrm{MA}$. It is understood that autophagy is accompanied by inhibition of the phospho-AKT (13) and inhibits phosphorylation of mTOR, as rapamycin induces autophagy by inhibiting mTOR (19). However, our results 

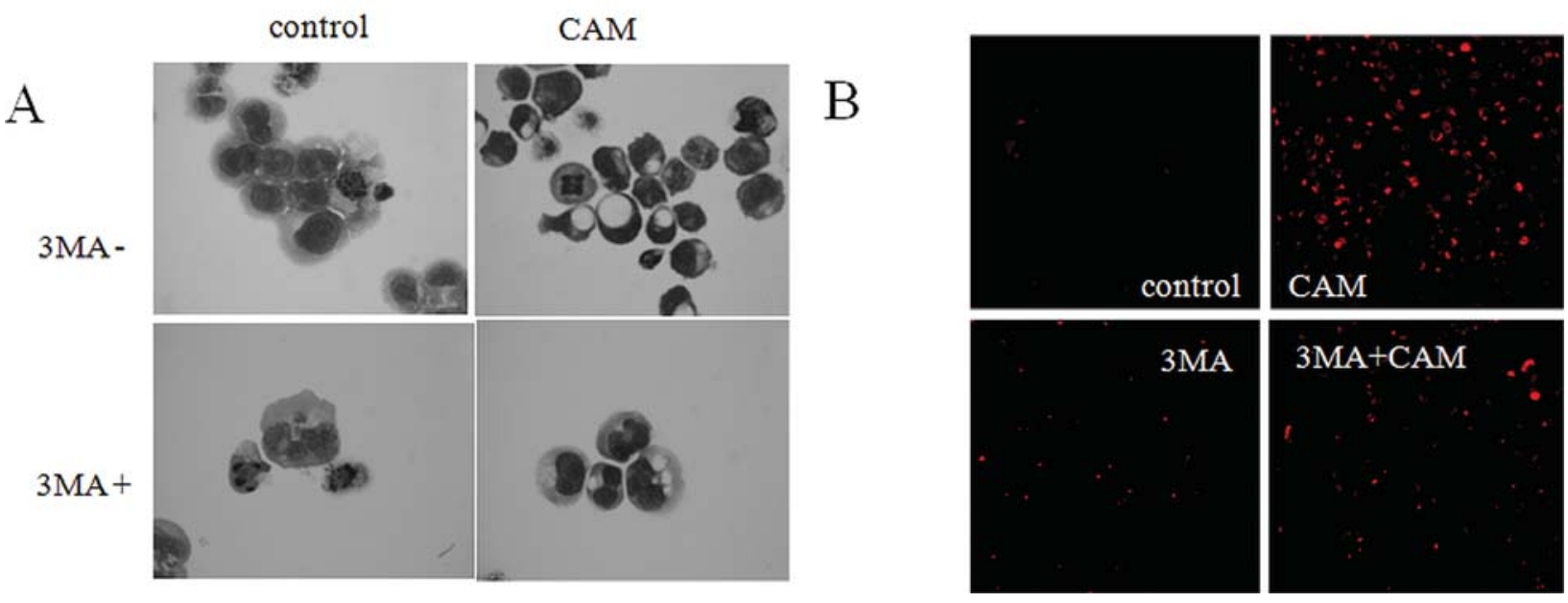

Figure 8. Inhibition of CAM-induced vacuole formation by 3-methyladenine (3MA). 12PE cells were treated with CAM at $50 \mu \mathrm{g} / \mathrm{ml}$ with or without $3 \mathrm{MA}$. (A) May-Giemsa staining. (B) LysoTracker staining. In the presence of 3MA, there was significant loss of vacuoles.
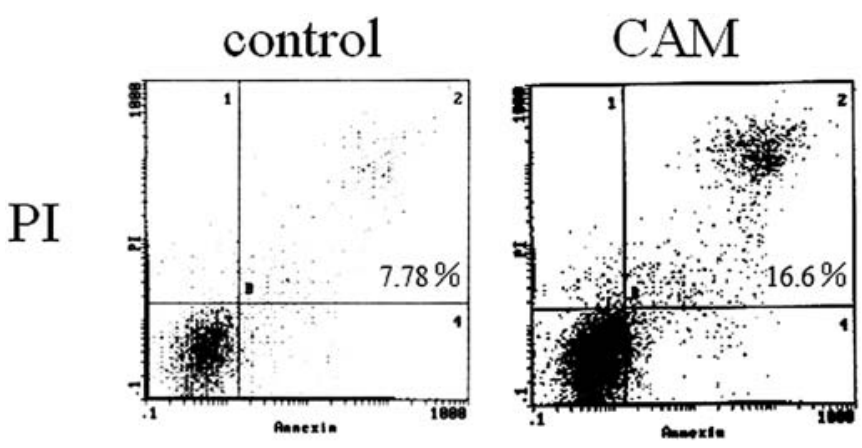

\section{AnnexinV}

Figure 9. Induction of cell death by CAM. 12PE cells were treated with CAM at $50 \mu \mathrm{g} / \mathrm{ml}$ for $18 \mathrm{~h}$. Cells were then stained with Annexin V/PI and analyzed with flow cytometry. Induction of cell death, which is characterized by both Annexin V- and PI-positive cells, was found in CAM-treated cells (right panel). showed that CAM did not inhibit phosphorylation of AKT nor p70S6K, indicating that CAM does not likely induce autophagy.

Surprisingly, there was accumulation of large vacuoles in a time-dependent manner in the presence of CAM, even $18 \mathrm{~h}$ after its addition. This finding might be explained by potent and continuous inhibition of autophagy by CAM at late stage of autophagy process. As we found marked accumulation of LC3-II by the treatment with bafilomycin (data not shown), CAM may exert similar effect although the point of inhibition may be different (Fig. 10). Based on the fact that both bafilomycin and CAM belong to macrolides, inhibition of autophagy process by CAM may not be an unexpected phenomenon. It is hypothesized that accumulation of autolysosomes is needed for CAM to encapsulate foreign microorganisms into autophagosomes, since autophagy is not only an energy-recycling process, but also a self-defense process, as shown with Streptococcus (20). This hypothesis may fit our findings, because CAM is an active agent against

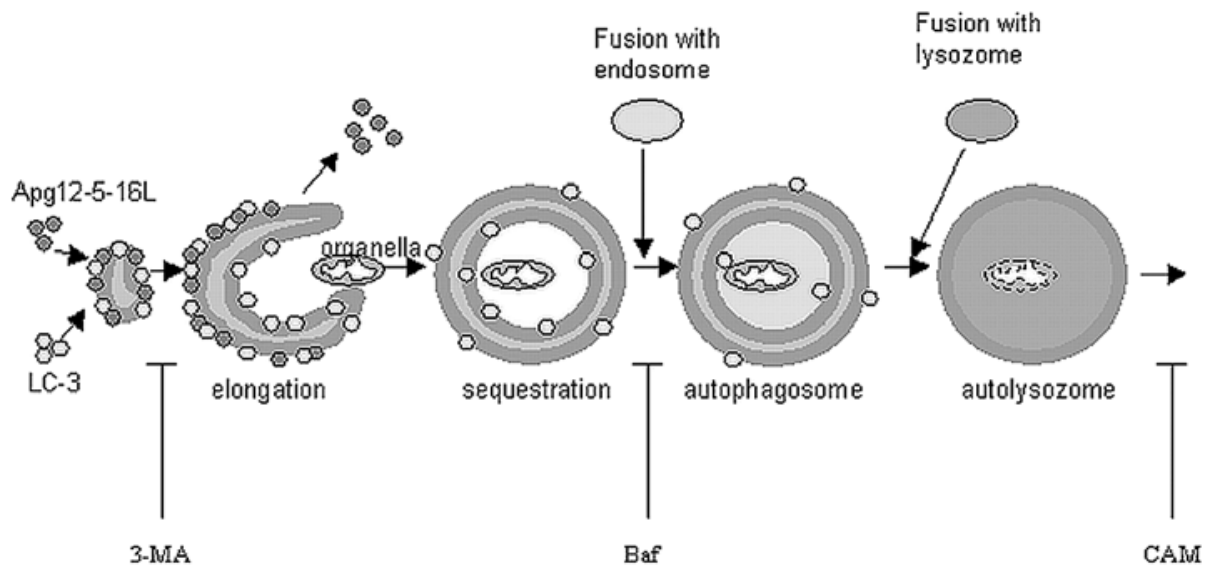

Figure 10. A hypothesis explaining inhibition of autophagy process by CAM. The autophagy process is inhibited by 3-methyladenine (3-MA) and bafilomycin (Baf) at early point and fusion with autophagosomes, respectively. Our results suggest that CAM may inhibit the process at late stage of autophagy. 
intracellular micro-orgasms such as Mycobacterium. Despite the fact that we found accumulation of autophagic vacuoles by CAM in tumor cells, a similar phenomenon may exist in normal immune cells, such as macrophages. Further evaluation of the hypothesis requires an experimental system that utilizes microorganisms and macrophages.

We found a cytotoxic effect of CAM in myeloma cells. This effect is distinct from apoptosis in terms of characteristics in morphology, flow cytometry analysis and activation of caspases. We consider that CAM-induced cell death might be from inhibition of autophagy. It is hypothesized that autophagy might be constitutively active in myeloma cells because of their hypoxic circumstances in the bone marrow where myeloma cells grow in vivo as Azad et al reported (21). Therefore, autophagy should play an important role in myeloma cells under various stresses (22). CAM may disrupt this rescue pathway thus resulting in cell death in vivo as we found vacuole formations in primary MM cells. This concept has been reported by Ito et al, showing inhibition of autophagy results various anti-tumor effects in malignant tumors $(23,24)$.

We found that CAM induced various phenomenon at concentration from 6 to $50 \mu \mathrm{g} / \mathrm{ml}$. Pharmacokinetic analysis showed concentration of CAM in serum is approximately $1 \mu \mathrm{g} / \mathrm{ml}$ (25) while it concentrates $>10$ times in cytoplasm (26), indicating that our in vitro experiments were appropriate.

We believe that this report is the first showing induction of autophagy by CAM, which is a well-known and worldwide utilized orally taken antibiotic. Despite our findings only referring to myeloma cells, a similar phenomenon may be found in various tumor cells, which show a survival benefit following treatment with CAM, such as lung cancer cells $(1-5,27,28)$. In this context, unveiling of mechanisms regulating autophagy by CAM should be important not only for understanding the biology of cancer cells but also extending survival of cancer patients.

\section{Acknowledgements}

Authors appreciate the useful suggestions from Dr Akitsugu Yamamoto (Department of Cell Biology, Faculty of BioScience, Nagahama Institute of Bio-Science and Technology, Shiga, Japan).

\section{References}

1. Mikasa K, Sawaki M, Kita E, et al: Significant survival benefit to patients with advanced non-small-cell lung cancer from treatment with clarithromycin. Chemotherapy 43: 288-296, 1997.

2. Sassa K, Mizushima Y, Fujishita T, Oosaki R and Kobayashi M: Therapeutic effect of clarithromycin on a transplanted tumor in rats. Antimicrob Agents Chemother 43: 67-72, 1999.

3. Hamada K, Mikasa K, Yunou Y, et al: Adjuvant effect of clarithromycin on chemotherapy for murine lung cancer. Chemotherapy 46: 49-61, 2000.

4. Mikasa K: Study of effects and mechanisms of macrolide as biological response modifier for lung cancer treatment. Jpn J Antibiot 53: 253-260, 2000.

5. Sakamoto M, Mikasa K, Majima T, et al: Usefulness of clarithromycin in patients with unresectable non-small-cell lung cancer-clinical evaluation of those who survived for a long time and those succumbed in a short period. Jpn J Antibiot 53 (Suppl. A): S56-S59, 2000.
6. Yatsunami J, Turuta N, Wakamatsu K, Hara N and Hayashi S: Clarithromycin is a potent inhibitor of tumor-induced angiogenesis. Res Exp Med (Berl) 197: 189-197, 1997.

7. Inoue K, Sakata K, Shibazaki M, Nishi Y, Sakamoto Y and Matsuo $\mathrm{H}$ : Inhibitory effects of macrolide antibiotics on infiltration and proliferation of lung cancer cell lines, A-549 and SBC-3. Jpn J Antibiot 51 (Suppl. A): S79-S80, 1998.

8. Coleman M, Leonard J, Lyons L, et al: Blt-d (clarithromycin [Biaxin], low-dose thalidomide, and dexamethasone) for the treatment of myeloma and Waldenstrom's macroglobulinemia. Leuk Lymphoma 43: 1777-1782, 2002.

9. Musto P, Falcone A, Sanpaolo G, Bodenizza C, Carotenuto M and Carella AM: Inefficacy of clarithromycin in advanced multiple myeloma: A definitive report. Haematologica 87: 658-659, 2002.

10. Niesvizky R, Jayabalan DS, Christos PJ, et al: BiRD (Biaxin [clarithromycin]/Revlimid [lenalidomide]/dexamethasone) combination therapy results in high complete- and overallresponse rates in treatment-naive symptomatic multiple myeloma. Blood 111: 1101-1109, 2008.

11. Apel A, Zentgraf H, Buchler MW and Herr I: Autophagy - a double-edged sword in oncology. Int J Cancer 125: 991-995, 2009.

12. Ohtsuki T, Yawata Y, Wada H, Sugihara T, Mori M and Namba M: Two human myeloma cell lines, amylase-producing KMS-12-PE and amylase-non-producing KMS-12-BM, were established from a patient, having the same chromosome marker, t(11;14)(q13;q32). Br J Haematol 73: 199-204, 1989.

13. Ellington AA, Berhow MA and Singletary KW: Inhibition of Akt signaling and enhanced ERK1/2 activity are involved in induction of macroautophagy by triterpenoid B-group soyasaponins in colon cancer cells. Carcinogenesis 27: 298-306, 2006.

14. Radimerski T, Montagne J, Hemmings-Mieszczak M and Thomas G: Lethality of Drosophila lacking TSC tumor suppressor function rescued by reducing dS6K signaling. Genes Dev 16: 2627-2632, 2002.

15. Klionsky DJ, Meijer AJ and Codogno P: Autophagy and p70S6 kinase. Autophagy 1: 59-61, 2005.

16. Swanson MS: Autophagy: Eating for good health. J Immunol 177: 4945-4951, 2006.

17. Mizushima N: Methods for monitoring autophagy. Int J Biochem Cell Biol 36: 2491-2502, 2004.

18. Lindmo K and Stenmark H: Regulation of membrane traffic by phosphoinositide 3-kinases. J Cell Sci 119: 605-614, 2006.

19. Easton JB and Houghton PJ: mTOR and cancer therapy. Oncogene 25: 6436-6446, 2006.

20. Nakagawa I, Amano A, Mizushima N, et al: Autophagy defends cells against invading group a streptococcus. Science 306: 1037-1040, 2004.

21. Azad MB, Chen Y, Henson ES, et al: Hypoxia induces autophagic cell death in apoptosis-competent cells through a mechanism involving BNIP3. Autophagy 4: 195-204, 2008.

22. Mathew R, Karantza-Wadsworth V and White E: Role of autophagy in cancer. Nat Rev Cancer 7: 961-967, 2007.

23. Ito S, Koshikawa N, Mochizuki S and Takenaga K: 3methyladenine suppresses cell migration and invasion of HT1080 fibrosarcoma cells through inhibiting phosphoinositide 3-kinases independently of autophagy inhibition. Int J Oncol 31: 261-268, 2007.

24. Ito H, Daido S, Kanzawa T, Kondo S and Kondo Y: Radiationinduced autophagy is associated with LC3 and its inhibition sensitizes malignant glioma cells. Int J Oncol 26: 1401-1410, 2005.

25. Yoshida H and Furuta T: Tissue penetration properties of macrolide antibiotics-comparative tissue distribution of erythromycinstearate, clarithromycin, roxithromycin and azithromycin in rats. Jpn J Antibiot 52: 497-503, 1999.

26. Tulkens PM: Intracellular distribution and activity of antibiotics. Eur J Clin Microbiol Infect Dis 10: 100-106, 1991.

27. Sakamoto M, Mikasa K, Hamada K, et al: Effect of clarithromycin treatment of natural killer cell activity in patients with advanced non-small cell lung cancer. Gan To Kagaku Ryoho 25: 2259-2266, 1998.

28. Majima T, Mikasa K, Sakamoto M, et al: Administration of clarithromycin (CAM) to non-small cell lung cancer. Jpn J Antibiot 53 (Suppl. A): S52-S55, 2000. 